\title{
A Rare Form of Mesothelioma: Malignant Pleural Deciduoid Mesothelioma
}

\author{
Timuçin Alar¹, Cemal Özçelik², Nihal Kılınç3, Ufuk Yılmaz ${ }^{4}$
}

\author{
${ }^{1}$ Department of Thoracic Surgery, Çanakkale Onsekiz Mart University Faculty of Medicine, Çanakkale \\ ${ }^{2}$ Department of Thoracic Surgery, Çukurova University Faculty of Medicine, Adana \\ ${ }^{3}$ Department of Pathology, Çanakkale Onsekiz Mart University Faculty of Medicine, Çanakkale \\ ${ }^{4}$ Chest Diseases Clinic, Dr. Suat Seren Chest Diseases and Surgery Training and Research Hospital, İzmir
}

\begin{abstract}
Mesothelioma usually develops in elder male patients that are exposed to asbestos. Deciduoid mesothelioma is a rare variant of epithelioid mesothelioma and has a poorer prognosis than epithelioid mesothelioma. As this subtype was seen in the peritoneum of young females and its relation with asbestos was not definite, these lesions were formerly thought to be stimulated by endogenous hormones. In the subsequent years, a relation was established between this tumor and asbestos since it was seen in the pericardium and pleura of males. In this paper, we present a 64-year-old male patient with asbestos exposure, diagnosed as having malignant pleural deciduoid mesothelioma, in which a long survival was provided with chemotherapy after surgery.
\end{abstract}

Keywords: Mesothelioma, asbestosis, surgery

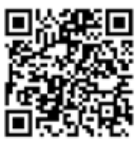

Received date: 16.04.2012 Accepted date: 07.07.2012

\section{Address for correspondence}

Timuçin Alar, Department of Thoracic Surgery, Çanakkale Onsekiz Mart University Faculty of

Medicine, Çanakkale, Turkey

E-mail: timalar@comu.edu.tr

(C) Copyright 2014 Turkish Respiratory Society (TRS) DOI: 10.5152/ejp.2014.80774

-Available online at www.eurasianjpulmonol.com

\section{INTRODUCTION}

Deciduoid mesothelioma is a rare variant of epithelioid mesothelioma. It was first defined in the peritoneum of a young female, and as asbestos exposure was not present in the history of that patient, asbestos mineral has not been considered to play a role in the aetiology of deciduoid mesothelioma (1). The fact that it was reported particularly in young females led to the consideration that it might be associated with endogenous hormones. However, as the tumour was identified in the pericardium and pleura of males in the subsequent years, this consideration is no longer valid (2). It is known that histological differentiation of mesothelioma from adenocarcinoma is difficult. Therefore, accurate diagnosis of deciduoid mesothelioma, which has a poorer prognosis than epithelioid mesothelioma, is important in guiding the treatment (3).

In this paper, we presented a male patient, who was diagnosed with malignant pleural deciduoid mesothelioma, was performed pleuropneumonectomy, and was followed up for one year after postoperative chemotherapy without any relapses.

\section{CASE PRESENTATION}

A 64-year-old male patient was admitted to our clinic with shortness of breath and right chest pain. His anamnesis revealed no additional complaints such as cough, fever or weight loss. His physical examination was unremarkable except for decreased breath sounds in the right middle and lower zones. On his postero-anterior chest $x$-ray, a homogenous density enhancement, which obscured the right costophrenic angle and rose to the level of the anterior end of the $3^{\text {rd }}$ rib with a parabolic contour, was observed (Figure 1). No culture growth, including tuberculosis, was identified in the pleural fluid obtained by thoracentesis. However, cytological examination of the pleural fluid demonstrated atypical mesothelial cell groups in a lymphocyte-rich background and it was reported that a tissue sample was required for 
definite diagnosis. Considering that high levels of pleural fluid would hinder a potential pulmonary mass, computed tomography (CT) of thorax was not performed. In April 2011, the patient underwent pleural biopsy via right videothoracoscopy (VATS). On exploration that was performed during videothoracoscopy after draining 2500 cc of fluid, patchy irregular thickening and various sizes of nodules changing between 2 and $6 \mathrm{~mm}$ were identified in the parietal pleura. Postoperative thoracic CT demonstrated nodular infiltrations in the pulmonary parenchyma (Figure 2). Histopathological examination of the specimen was reported as "malignant deciduoid mesothelioma". Accordingly, a detailed anamnesis revealed that he had lived in a village until the age of 20 years and had been exposed to asbestos mineral. Positron Emission Tomography (PET-CT) and Magnetic resonance Imaging (MRI) of brain were performed to explore the presence of potential metastasis and no distant metastasis was detected (Figure 3). As mediastinal lymph node involvement was not detected in PET-CT, preoperative mediastinoscopy was not performed. In positron emission tomography, nodular changes characterized by 18-Fluoro-deoxy-glucose (FDG) uptake were reported in the mediastinum, costo-diaphragmatic pleura and fissures and loculated pleural effusion was identified in the right hemithorax (primarily in favour of mesothelioma). Pulmonary function tests and cardiac assessment revealed that the patient could tolerate a major surgery such as pleuropneumonectomy. Moreover, it was concluded that this surgery would be more convenient as the histopathological type of the tumour was epithelioid and invasion of both fissures was detected in PET scan. Following necessary preparations, thoracic cavity was opened after resection of the right $6^{\text {th }}$ rib. Observing the invasion of the fissure, as was mentioned in the report of PET-CT, pleuropneumonectomy with partial resection of the diaphragm and pericardium were performed and polytetrafluoroethylene (PTFE) grafts were used for reconstruction. After the patient was followed up in the intensive care unit for one day, he was transferred to the ward and was discharged from the hospital on the postoperative $6^{\text {th }}$ day. Histopathological examination of the material was reported as follows: "The tumour involves entire pleura and infiltrates the lung. No tumour is seen on the rib. The resected 15 lymph nodes $(2 R=4,4 R=6$ and around the main bronchus $=5$ ) are reactive. No tumour in bronchi and surgical border". Therefore, consultation was requested from the oncology clinic and the patient was started on chemotherapy 3 months after the surgery (Augusts 2011). The patient received 4 cycles of cisplatinum $\left(75 \mathrm{mg} / \mathrm{m}^{2}\right)$ and pemetrexed disodium $\left(500 \mathrm{mg} / \mathrm{m}^{2}\right)$, and then followed up. No relapse was determined on his examinations including Thoracic $\mathrm{CT}$, Brain MRI, and PET-CT on the postoperative $1^{\text {st }}$ year. Consent was obtained from the patient for writing this case report.

\section{DISCUSSION}

The pleura, which is involved by secondary tumours rather than primary tumours, is a membrane, in which lung and breast cancer metastases are detected most frequently. Mesothelioma arises from multipotent mesothelial or subserosal cells that are likely to produce epithelioid or sarcomatoid cancers. As is known, it is difficult to differentiate mesothelioma, which is more prevalent among advanced aged males with a history of asbestos exposure, from metastatic adenocarcinoma (4). Diagnosis of mesothelioma is made by immunohistochemical examinations; deciduoid type, a variant of epithelioid type, is rarely seen. Less than $50 \%$ of this type detected in less than 50 cases has been reported in the pleura (3). Immunostaining techniques used in the differential diagnosis include pancytokeratin, cytokeratin (CK) 5/6, calretinin and Wilms tumor (WT1). While epithelioid type is strongly stained with pancytokeratin, CK $5 / 6$ is beneficial in the differentiation of mesothelioma

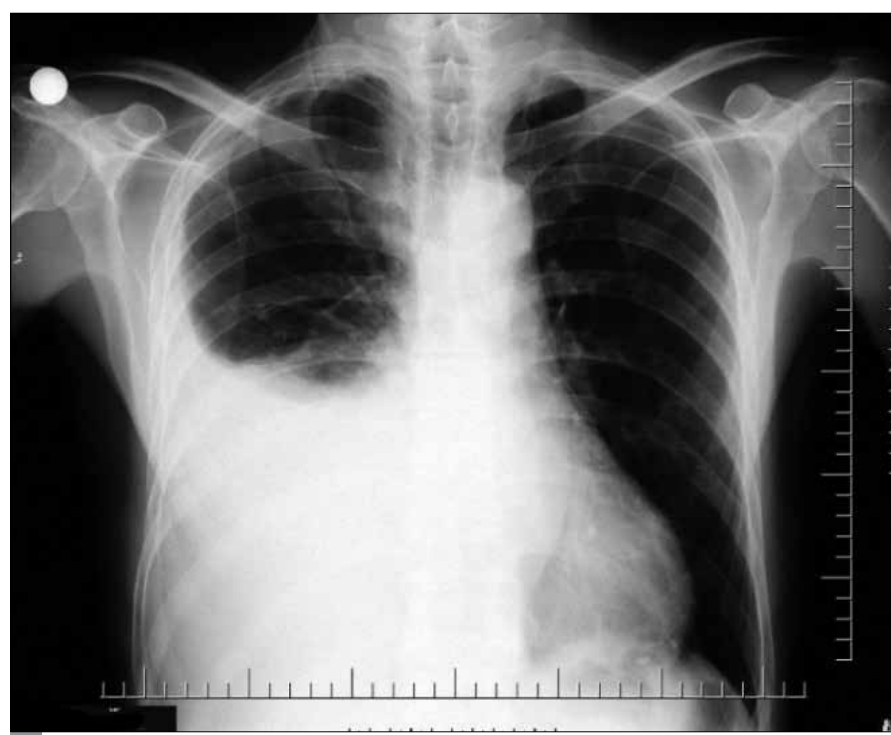

Figure 1. PA chest $x$-ray at the time of admission of the patient

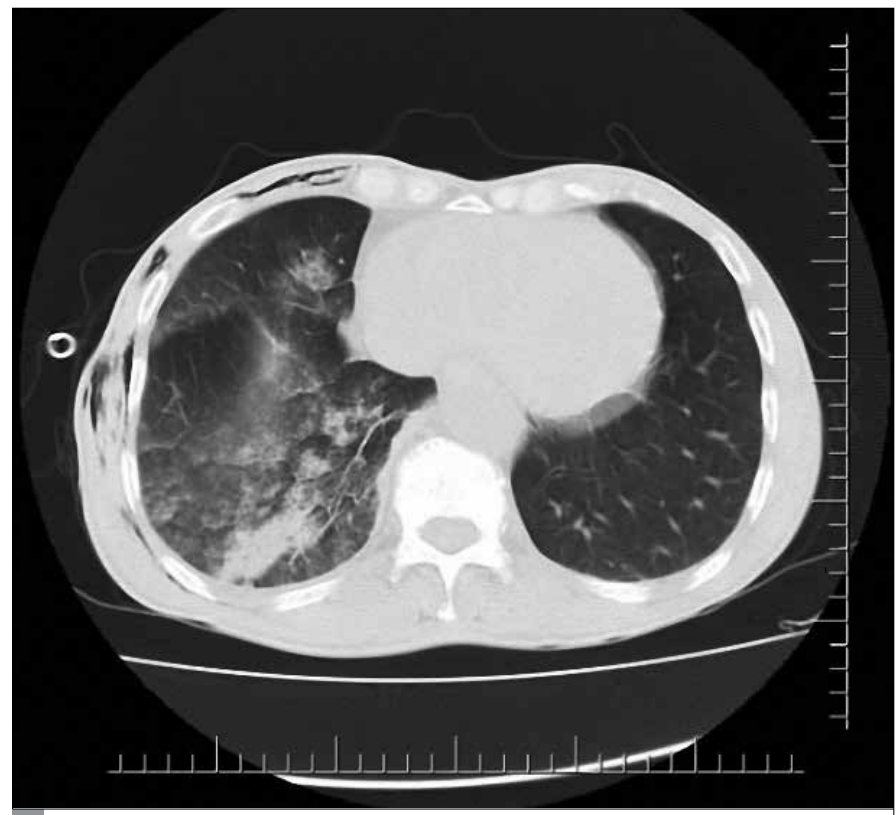

Figure 2. Computed tomography of Thorax after videothoracoscopic effusion drainage and pleural biopsy

from pulmonary adenocarcinoma. Calretinin is the best known and the most specific marker of mesothelioma and positive nuclear staining is only seen in mesothelioma (5). The present case was differentiated from metastatic adenocarcinoma by detecting positive "anti-calretinin antibodies" in the staining of the specimens obtained by thoracoscopic biopsy. In addition, identification of deciduoid cells with large eosinophylic cytoplasm and strongly positive anti-nuclear calretinin antibodies made the diagnosis definite (Figure 3 ).

It is known that asbestos mineral plays a role in the aetiology of mesothelioma. However, initially, such a relation could not be established with the deciduoid subtype, but thereafter a probable relationship with asbestos has been considered based on reported papers (6). When the anamnesis of the present case was detailed, we as well learned that he was exposed to asbestos mineral, which is used in 


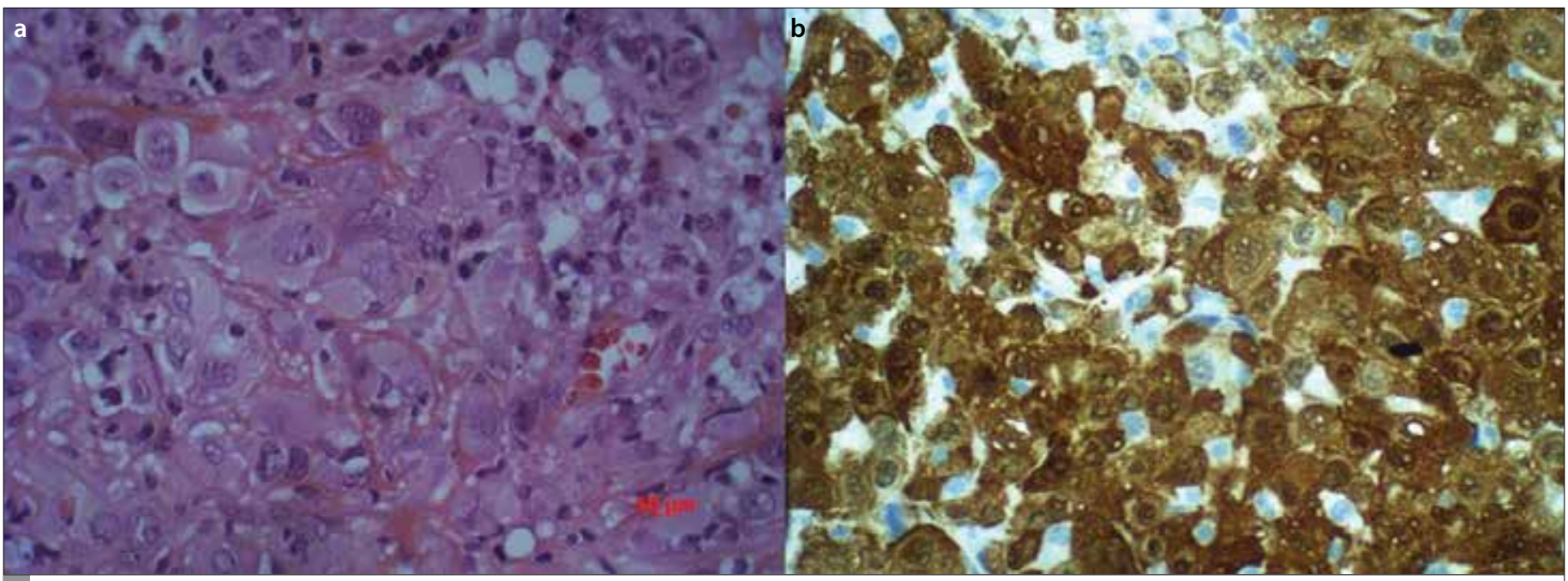

Figure 3. a, b. Deciduoid-like tumour cells with large eosinophilic cytoplasm and prominent nucleoli (Haematoxylin-eosin x400) (a) Anti cytoplasmic and strong anti-nuclear antibody positivity against calretinin in tumour cells (x400) (b)

the rural areas in Turkey for in-house whitewash instead of lime and known as "white soil" and contains asbestos mineral, for his house was in the Ovacık village of Biga county of Çanakkale, where he had lived until the age of 20 years. The mean time-span for development of asbestos-induced mesothelioma is reported between 14 and 72 years (7). In the present case, the disease appeared approximately 40 years later, which is consistent with the literature.

Mean survival reported for deciduoid mesothelioma, which is more aggressive as compared to epithelioid mesothelioma, is 9.6 months (1, 2). However, there are authors reporting cases that lived for a mean of 23 months (17-39 months) only with chemotherapy or symptomatic treatment without surgery (8). Nevertheless, authors could not explain such a long survival and recommended investigation of new cases. In the present case, we made the definite diagnosis by thoracoscopic biopsy, which was performed due to the failure in making diagnosis via thoracentesis, and performed systemic screening. The present patient, who had no metastasis received chemotherapy after radical surgery, and is being followed up for 12 months with no relapse.

Radical surgery in malignant mesothelioma is currently a debatable issue. Because of the absence of randomized studies, the Mesothelioma and Radical Surgery (MARS) study did not report a definite judgment about the surgical method that is superior (pleurectomy or extrapleural pneumonectomy) (9). We performed extrapleural pneumonectomy since we detected fissure on PET-CT and during surgery. Moreover, preoperative evaluation of the patient revealed that he could tolerate this radical surgery.

\section{CONCLUSION}

Malign deciduoid mesothelioma, which could be detected in both genders, could involve both the pleura and the peritoneum. It is a variant of epithelioid mesothelioma, has a poorer prognosis and its relation with asbestos is being increasingly detected. Along with the case presented herein, we think that combination of radical surgery and chemotherapy may contribute to longer survival and reduce relapses in this subtype of mesothelioma, and that the etiopathogenesis of this tumour will be enlightened more along with immunohistochemical studies.
Informed Consent: Written informed consent was obtained from patients who participated in this study.

Peer-review: Externally peer-reviewed.

Author contributions: Concept - T.A., C.Ö., N.K., U.Y.; Design - T.A., C.Ö., N.K., U.Y.; Supervision - T.A, C.Ö., N.K., U.Y.; Resource - N.K., U.Y.; Materials - T.A., U.Y., N.K.; Data Collection\&/or Processing - T.A., C.Ö., N.K.,U.Y.; Analysis\&/or Interpretation - T.A., U.Y.; Literature Search - T.A., C.Ö.; Writing - T.A., U.Y., C.Ö.; Critical Reviews - T.A., C.Ö., N.K., U.Y.

Conflict of Interest: No conflict of interest was declared by the authors.

Financial Disclosure: The authors declared that this study has received no financial support.

\section{REFERENCES}

1. Nascimento AG, Keeney GL, Fletcher CD. Deciduoid peritoneal mesothelioma. An unusual phenotype affecting young females. Am J Surg Pathol 1994; 18: 439-45. [CrossRef]

2. Puttagunta L, Vriend RA, Nguyen GK. Deciduoid epithelial mesothelioma of the pleura with focal rhabdoid change. Am J Surg Pathol 2000; 24: 1440-3. [CrossRef]

3. Santos C, Gamboa F, Fradinho F, Pego A, Carvalho L, Bernardo J. Deciduoid pleural mesothelioma - a rare entity in a young woman. Rev Port Pneumol 2012; 18: 294-8. [CrossRef]

4. Gloeckner-Hofmann K, Zhu XZ, Bartels H, Feller AC, Merz H. Deciduoid pleural mesothelioma affecting a young female without prior asbestos exposure. Respiration 2000; 67: 456-8. [CrossRef]

5. Ordonez NG. Immunohistochemical diagnosis of epithelial mesothelioma: an update. Arch Pathol Lab Med 2005; 129: 1407-14.

6. Monaghan $\mathrm{H}, \mathrm{Al}-\mathrm{Nafussi} \mathrm{A}$. Deciduoid pleural mesothelioma. Histopathology 2001; 39: 104-6. [CrossRef]

7. Bianchi C, Giarelli L, Grandi G, Brollo A, Ramani L, Zuch C. Latency periods in asbestos-related mesothelioma of the pleura. Eur J Cancer Rev 1997; 6: $162-6$

8. Serio G, Scattone A, Pennella A, Giardina C, Musti M, Valente T, et al. Malignant deciduoid mesothelioma of the pleura: report of two cases with long survival. Histopathology 2002; 40: 348-52. [CrossRef]

9. Treasure T, Waller D, Tan C, Entwisle J, O'Brien M, O'Byrne K, et al. The mesothelioma and radical surgery randomized controlled trial: the MARS feasibility study. J Thorac Oncol 2009; 4: 1254-58. [CrossRef] 